\title{
The prevalence and risk factors on different degrees of non-alcoholic fatty liver disease in Shanghai
}

\author{
Xiao-na Hu \\ Huadong Hospital Affiliated to Fudan University \\ Yi-qin Huang \\ Huadong Hospital Affiliated to Fudan University \\ Jiao-feng Wang \\ Huadong Hospital Affiliated to Fudan University \\ Fang-yuan Dong \\ Huadong Hospital Affiliated to Fudan University \\ Xiao-feng Yu \\ Huadong Hospital Affiliated to Fudan University \\ Jie Chen \\ Huadong Hospital Affiliated to Fudan University \\ Zhi-jun Bao ( $\nabla$ xinyi8681@sina.com ) \\ Huadong Hospital Affiliated to Fudan University
}

Research article

Keywords: Prevalence, risk factors, non-alcoholic fatty liver disease

Posted Date: June 1st, 2020

DOI: https://doi.org/10.21203/rs.3.rs-30325/v1

License: () (1) This work is licensed under a Creative Commons Attribution 4.0 International License. Read Full License 


\section{Abstract}

\section{[Background and aims]}

NAFLD is a common chronic liver disease. The purpose of this study is to carry out a current large-scale epidemiological survey and analyse factors related to the incidence and the severity of NAFLD.

\section{[Methods]}

Three communities of Shanghai were selected by stratified random sampling in 2019.

The residents older than 18 years old underwent medical history taking, physical examination, laboratory examination and liver ultrasonography examination. The prevalence of different degrees of NAFLD, the prevalence of metabolic factors, and factors associated with the severity of NAFLD was analysed.

\section{[Results]}

A total of 19250 subjects were enrolled. The total prevalence of NAFLD in Shanghai was $45.55 \%, 62.00 \%$ in males and $27.54 \%$ in females. Age, FLI, BMI, WC, ALT, AST, AKP, GGT, CR, FPG, UA, TC, TG and LDL in NAFLD patients were higher than those in non-NAFLD people. The distribution of NAFLD prevalence was different in different age groups. The prevalence of diabetes, obesity and hypertriglyceridemia in the NAFLD group were higher than that in the non-NAFLD group. In males, FLI, weight, WC, ALT, UA, TG, GGT and metabolic syndrome were positively correlated with the severity of NAFLD; while HDL was negatively correlated with it. In females, age, FLI, weight, WC, ALT, FPG, UA, TG, LDL, GGT, AKP and metabolic syndrome were positively correlated with the severity of NAFLD; while HDL was negatively correlated with it.

\section{[Conclusions]}

The prevalence of NAFLD in Shanghai is high. The distribution of NAFLD prevalence was different in different age groups. Many factors are related to the incidence of NAFLD and the severity of NAFLD.

\section{Introduction}

Non-alcoholic fatty liver disease (NAFLD) is a clinicopathological syndrome characterized by excessive fat deposition in hepatocytes in the absence of chronic viral hepatitis, significant alcohol consumption, drug-induced liver injury, autoimmune hepatitis and other chronic liver diseases such as or Wilson's disease. NAFLD consists of a wide spectrum of diseases, including non-alcoholic fatty liver (NAFL), non-alcoholic steatohepatitis (NASH), and NASH-related liver fibrosis and cirrhosis, and can even progress to liver carcinoma or liver failure, posing a serious threat to human health[1]. The prevalence of NAFLD varies in different regions. The global prevalence of NAFLD is $25.24 \%$. The highest prevalence of NAFLD is in the Middle East and South America, with rates of $31.79 \%$ and $30.45 \%$, respectively, while the lowest prevalence of NAFLD is in Africa, with a rate of $14 \%$. However, the prevalence of NAFLD in Asia is approximately 27.37\% which has surpassed that in Europe (23.71\%)[2]. Some epidemiological investigations have been conducted in China in recent years. The average prevalence of NAFLD in China was approximately 20.1\% in 2014[3]. The prevalence of NAFLD in males was approximately $43.3 \%$ in Shanghai in 2016[4]. Our previous study showed that the total prevalence of NAFLD was 38.17\% in the Shanghai working population in 2012[5]. However, the latest prevalence of NAFLD in the whole population in Shanghai is unknown.

NAFLD is closely related to metabolic syndrome such as hypertension, type 2 diabetes, coronary heart disease and colorectal cancer. Therefore, we conducted a large-scale survey to investigate the epidemiological status of NAFLD, and to analyse the risk factors related to the severity of NAFLD which might have important clinical significance for the prevention, early diagnosis and treatment of NAFLD.

\section{Materials And Methods Subject recruitment}

The subjects of this study were permanent residents in Shanghai from June 2019 to September 2019. We randomly selected three districts from 16 districts in Shanghai and then selected three communities by a cluster random sampling method. The permanent residents over 18 years old in the community were taken as the research subjects. The study was approved by the Research Ethics Committee of Huadong Hospital, Fudan University. All the subjects signed the informed consent form for blood samples or tissue samples to be used in clinical experiments.

\section{History taking}

Questionnaires were used to collect information on sex, age, education, occupation, past history of diseases, infectious diseases such as hepatitis and tuberculosis, smoking history, drinking history and daily alcohol consumption, medication status, history of rheumatic immune diseases and family history of diseases.

\section{Physical examination}


All participants fasted overnight before the medical examination. Blood pressure (BP), weight, height and waist circumference (WC) were measured by a trained examiner. Body mass index (BMI) was calculated as weight $(\mathrm{kg}) /$ stature $\left(\mathrm{m}^{2}\right)[5]$.

\section{Laboratory assessments}

Serum levels of alanine aminotransferase(ALT), aspartate aminotransferase(AST), alkaline phosphatase(AKP), gamma-glutamyl transferase(GGT), fasting plasma glucose(FPG), total cholesterol (TC), triglyceride(TG), low density lipoprotein cholesterol(LDL), high density lipoprotein cholesterol(HDL), creatinine (CR) and uric acid (UA) were measured by a multichannel autoanalyser. The participants with evidence of abnormal blood tests of liver function had further investigations performed including serology of hepatitis B and C, and autoimmune markers such as antinuclear antibody(ANA), antismooth muscle antibody(AMA), and antimito-chondrial antibody.

\section{Ultrasonographic examination}

Liver ultrasonography (US) was performed in all participants by five trained operators. The diagnosis of fatty liver meets the three criteria as follows: increased hepatic echogenicity, blurring of liver vasculature and deep attenuation of the ultrasonographic signal. NAFLD was divided into mild, moderate and severe fatty liver according to the ultrasonographic manifestations[5].

\section{Diagnostic criteria}

Patients with a clinical systolic blood pressure (SBP) $\geq 140 \mathrm{mmHg}$ and/or diastolic blood pressure (DBP) $\geq 90 \mathrm{mmHg}$ were defined as having hypertension[6]. FPG level $\geq 7.0 \mathrm{mmol} / \mathrm{L}$ was defined as diabetes, $5.6 \mathrm{mmol} / \mathrm{L} \leq \mathrm{FPG}<7.0 \mathrm{mmol} / \mathrm{L}$ was defined as pre-diabetes[7]. Metabolic syndrome was diagnosed following the criteria raised by the National Cholesterol Education Panel- Adult Treatment Panel III, and the modified guidelines in the Asia-Pacific region[8, 9]. The diagnostic criteria for NAFLD are as follows: negative tests for the presence of hepatitis B surface antigen and antibody to hepatitis $C$ virus; no drinking history or the average alcohol consumption less than $20 \mathrm{~g} / \mathrm{day}(140 \mathrm{~g} / \mathrm{w})$ in males and $10 \mathrm{~g} / \mathrm{day}$ (70 g/w) in females; no history of receiving or having recently received hepatotoxic drugs; no history of systematic illness known to cause fatty liver disease; no history of clinical, biochemical and US findings consistent with other chronic liver diseases, such as autoimmune liver disease, Wilson's disease or alpha-1antitrypsin deficiency[10, 11]. Fatty liver index (FLI) was used to predict the risk of NAFLD. FLI=

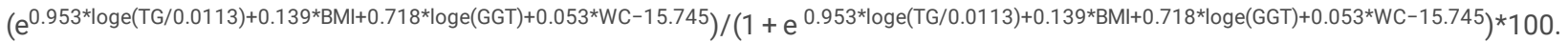

\section{Statistical Analyses}

Data were expressed as the mean \pm standard deviation (SD), inter-quartile range (IQR) or percentage. The $t$ test or rank sum test of two independent samples was used to compare the differences between the two groups in the measurement data, and the non-parametric test was used to compare the differences between two groups in the enumeration data. Chi-square test or Fisher's exact probability method was used to compare the prevalence between two or more groups. Spearman correlation analysis was used to analyzed the risk factors associated with the severity of NAFLD. All statistical analyses were performed using by SPSS 22.0. $P<0.05$ was considered a significant difference.

\section{Results}

\section{Characteristics of the participants}

A total of 19250 subjects were enrolled in this study, including 10062 males and 9188 females. A total of 8768 subjects were NAFLD patients, with a prevalence of $45.55 \%$. In the male population, 6238 subjects were NAFLD patients, accounting for $62 \%$. The age, FLI, height, weight, BMI, WC, ALT, AST, AKP, GGT, CR, FPG, UA, TC, TG and LDL indexes of the male NAFLD patients were significantly higher than those of non-NAFLD subjects $(P<0.05)$, while HDL in the NAFLD group was lower than that in the non-NAFLD group $(P<0.05)$. In the female population, 2530 subjects were NAFLD patients, accounting for $27.54 \%$. The age, FLI, height, weight, BMI, WC, ALT, AST, AKP, GGT, CR, FPG, UA, TC, TG and LDL indexes of the female NAFLD patients were significantly higher than those of non-NAFLD subjects $(P<0.05)$, while HDL in the NAFLD group was lower than that in the non-NAFLD group $(P<$ 0.05).(Table 1) 
Table 1

Differences between NAFLD patients and non-NAFLD subjects in males and females (mean \pm standard deviation)

Males

\begin{tabular}{|c|c|c|c|c|c|c|c|c|}
\hline & $\begin{array}{l}\text { Non-NAFLD } \\
(n=3824)\end{array}$ & $\begin{array}{l}\text { NAFLD } \\
(n=6238)\end{array}$ & $t / Z$ & $P$-value & $\begin{array}{l}\text { Non-NAFLD } \\
(\mathrm{n}=6658)\end{array}$ & $\operatorname{NAFLD}(\mathrm{n}=2530)$ & $t / Z$ & $P$-value \\
\hline Age(years) & $45.62 \pm 14.60$ & $49.17 \pm 13.30$ & 12.249 & $<0.001$ & $42.11 \pm 12.35$ & $53.15 \pm 13.25$ & 36.334 & $<0.001$ \\
\hline FLI & $\begin{array}{l}13.45(20.78- \\
31.71)\end{array}$ & $\begin{array}{l}30.94(48.15- \\
67.56)\end{array}$ & 53.954 & $<0.001$ & $\begin{array}{l}5.18(8.37- \\
14.11)\end{array}$ & $\begin{array}{l}16.70(26.91- \\
43.37)\end{array}$ & 53.345 & $<0.001$ \\
\hline Height (cm) & $171.76 \pm 6.39$ & $171.43 \pm 6.13$ & 2.528 & 0.012 & $160.04 \pm 6.03$ & $158.37 \pm 5.91$ & 11.918 & $<0.001$ \\
\hline Weight (kg) & $67.17 \pm 5.67$ & $77.57 \pm 10.47$ & 53.904 & $<0.001$ & $54.87 \pm 6.87$ & $64.58 \pm 9.12$ & 48.554 & $<0.001$ \\
\hline SBP (mmHg) & $121.87 \pm 16.85$ & $121.82 \pm 16.48$ & 0.152 & 0.879 & $120.86 \pm 16.83$ & $121.56 \pm 16.65$ & 1.800 & 0.072 \\
\hline $\mathrm{DBP}(\mathrm{mmHg})$ & $75.80 \pm 12.53$ & $75.95 \pm 12.26$ & 0.609 & 0.543 & $75.42 \pm 12.53$ & $75.40 \pm 11.99$ & 0.051 & 0.959 \\
\hline BMI & $23.17 \pm 3.20$ & $24.86 \pm 3.61$ & 24.418 & $<0.001$ & $22.81 \pm 3.38$ & $24.52 \pm 3.92$ & 19.405 & $<0.001$ \\
\hline WC (cm) & $88.09 \pm 1.38$ & $93.50 \pm 7.80$ & 53.516 & $<0.001$ & $79.35 \pm 2.17$ & $86.12 \pm 7.10$ & 47.139 & $<0.001$ \\
\hline $\operatorname{ALT}(\mathrm{U} / \mathrm{L})$ & $\begin{array}{l}13.00(17.00- \\
23.00)\end{array}$ & $\begin{array}{l}18.00(26.00- \\
38.00)\end{array}$ & 39.206 & $<0.001$ & $\begin{array}{l}9.00(11.00- \\
15.00)\end{array}$ & $\begin{array}{l}13.00(17.00- \\
26.00)\end{array}$ & 35.633 & $<0.001$ \\
\hline AST(U/L) & $\begin{array}{l}16.00(18.00- \\
22.00)\end{array}$ & $\begin{array}{l}17.00(21.00- \\
26.00)\end{array}$ & 23.006 & $<0.001$ & $\begin{array}{l}14.00(16.00- \\
19.00)\end{array}$ & $\begin{array}{l}15.00(18.00- \\
23.00)\end{array}$ & 21.366 & $<0.001$ \\
\hline $\operatorname{AKP}(\mathrm{U} / \mathrm{L})$ & $69.58 \pm 17.02$ & $72.36 \pm 17.84$ & 7.824 & $<0.001$ & $58.78 \pm 19.13$ & $72.16 \pm 20.96$ & 27.988 & $<0.001$ \\
\hline $\mathrm{GGT}(\mathrm{U} / \mathrm{L})$ & $\begin{array}{l}16.00(20.80- \\
29.70)\end{array}$ & $\begin{array}{l}24.10(33.90- \\
52.30)\end{array}$ & 27.272 & $<0.001$ & $\begin{array}{l}11.10(13.80- \\
18.10)\end{array}$ & $\begin{array}{l}15.50(21.10- \\
31.40)\end{array}$ & 37.064 & $<0.001$ \\
\hline $\mathrm{CR}(\mu \mathrm{mol} / \mathrm{L})$ & $84.64 \pm 11.14$ & $85.07 \pm 11.58$ & 2.478 & 0.013 & $62.77 \pm 11.05$ & $63.02 \pm 9.44$ & 1.099 & 0.272 \\
\hline $\mathrm{FPG}(\mathrm{mmol} / \mathrm{L})$ & $4.60(4.90-5.30)$ & $4.80(5.20-5.70)$ & 21.501 & $<0.001$ & $4.60(4.90-5.20)$ & $4.90(5.20-5.70)$ & 29.395 & $<0.001$ \\
\hline $\mathrm{UA}(\mu \mathrm{mol} / \mathrm{L})$ & $353.35 \pm 68.81$ & $393.60 \pm 76.74$ & 27.243 & $<0.001$ & $262.91 \pm 53.07$ & $306.67 \pm 65.67$ & 30.002 & $<0.001$ \\
\hline $\mathrm{TC}(\mathrm{mmol} / \mathrm{L})$ & $4.83 \pm 0.87$ & $5.23 \pm 0.96$ & 21.357 & $<0.001$ & $4.96 \pm 0.92$ & $5.44 \pm 1.00$ & 20.896 & $<0.001$ \\
\hline $\mathrm{TG}(\mathrm{mmol} / \mathrm{L})$ & $0.80(1.00-1.40)$ & $1.20(1.60-2.30)$ & 42.039 & $<0.001$ & $0.60(0.80-1.10)$ & $1.00(1.40-1.90)$ & 45.243 & $<0.001$ \\
\hline $\mathrm{HDL}(\mathrm{mmol} / \mathrm{L})$ & $1.46 \pm 0.28$ & $1.30 \pm 0.23$ & 30.127 & $<0.001$ & $1.71 \pm 0.31$ & $1.50 \pm 0.28$ & 31.183 & $<0.001$ \\
\hline LDL(mmol/L) & $2.70 \pm 0.69$ & $3.04 \pm 0.73$ & 23.956 & $<0.001$ & $2.62 \pm 0.71$ & $3.11 \pm 0.78$ & 27.651 & $<0.001$ \\
\hline
\end{tabular}

Table 2

Prevalence of NAFLD in different age groups of male and female population ( $\mathrm{n} / \%)$

\begin{tabular}{|c|c|c|c|c|c|c|c|c|c|c|}
\hline & Males & & & & & Females & & & & \\
\hline Age & $\begin{array}{l}\text { Non } \\
\text { NAFLD }\end{array}$ & $\begin{array}{l}\text { Mild } \\
\text { NAFLD }\end{array}$ & $\begin{array}{l}\text { Moderate } \\
\text { NAFLD }\end{array}$ & $\begin{array}{l}\text { Severe } \\
\text { NAFLD }\end{array}$ & Total & $\begin{array}{l}\text { Non } \\
\text { NAFLD }\end{array}$ & $\begin{array}{l}\text { Mild } \\
\text { NAFLD }\end{array}$ & $\begin{array}{l}\text { Moderate } \\
\text { NAFLD }\end{array}$ & $\begin{array}{l}\text { Severe } \\
\text { NAFLD }\end{array}$ & Total \\
\hline$<30$ & $450(64.8)$ & 159(22.9) & $83(12.0)$ & $2(0.3)$ & 694(100.0) & $846(94.0)$ & $40(4.4)$ & $14(1.6)$ & $0(0.0)$ & $900(100.0)$ \\
\hline $\begin{array}{l}{[30} \\
\sim 45\end{array}$ & $1645(41.2)$ & 1349(33.8) & $986(24.7)$ & 12(0.3) & 3992(100.0) & $3457(84.0)$ & 491(11.9) & $164(4.0)$ & $2(0.0)$ & $4114(100.0)$ \\
\hline $\begin{array}{l}{[45} \\
\tilde{60})\end{array}$ & $908(30.0)$ & $1236(40.8)$ & $870(28.8)$ & $12(0.4)$ & $3026(100.0)$ & $1589(62.6)$ & $727(28.7)$ & 219(8.6) & $2(0.1)$ & $2537(100.0)$ \\
\hline $\begin{array}{l}{[60} \\
\tilde{7} 5)\end{array}$ & $683(34.1)$ & 778(38.8) & $535(26.7)$ & $8(0.4)$ & $2004(100.0)$ & $655(46.5)$ & $511(36.3)$ & $240(17.1)$ & $2(0.1)$ & $1408(100.0)$ \\
\hline$\geq 75$ & 138(39.9) & $112(32.4)$ & $95(27.4)$ & $1(0.3)$ & $346(100.0)$ & $111(48.5)$ & $75(32.8)$ & $42(18.3)$ & $1(0.4)$ & $229(100.0)$ \\
\hline Total & $3824(38.0)$ & $3634(36.1)$ & $2569(27.5)$ & $35(0.3)$ & $1006(100.0)$ & $6658(72.5)$ & $1844(20.1)$ & $679(7.4)$ & $7(0.1)$ & $9188(100.0)$ \\
\hline
\end{tabular}


Table 3

Prevalence of metabolic syndromes in males and females $(\mathrm{n} / \%)$

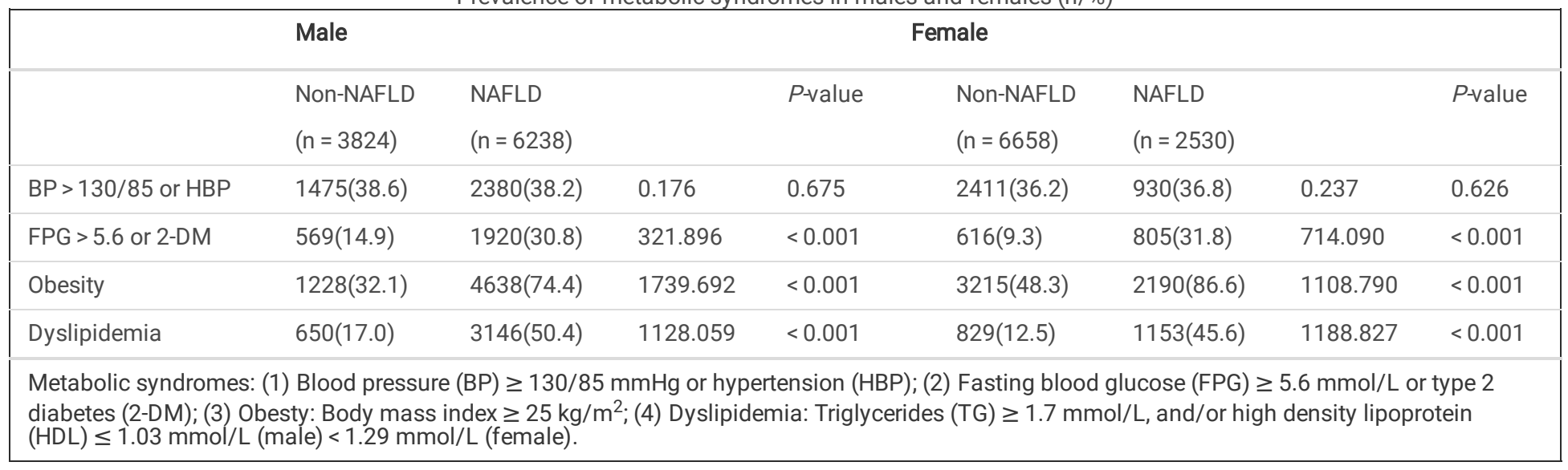

Table 4

Spearman analysis of correlative factors with the severity of NAFLD

\begin{tabular}{|c|c|c|c|c|}
\hline & \multicolumn{2}{|l|}{ male } & \multicolumn{2}{|l|}{ female } \\
\hline & Relative factors(r) & $P$-value & Relative factors(r) & $P$-value \\
\hline Age & 0.128 & $<0.001$ & 0.363 & $<0.001$ \\
\hline FLI & 0.605 & $<0.001$ & 0.567 & $<0.001$ \\
\hline Height & -0.010 & 0.314 & -0.127 & $<0.001$ \\
\hline Weight & 0.531 & $<0.001$ & 0.502 & $<0.001$ \\
\hline SBP & -0.002 & 0.840 & 0.020 & 0.050 \\
\hline DBP & -0.000 & 0.972 & 0.005 & 0.663 \\
\hline BMI & 0.266 & $<0.001$ & 0.209 & $<0.001$ \\
\hline WC & 0.507 & $<0.001$ & 0.545 & $<0.001$ \\
\hline ALT & 0.471 & $<0.001$ & 0.388 & $<0.001$ \\
\hline AST & 0.298 & $<0.001$ & 0.237 & $<0.001$ \\
\hline CR & -0.029 & 0.003 & 0.005 & 0.610 \\
\hline FPG & 0.240 & $<0.001$ & 0.313 & $<0.001$ \\
\hline UA & 0.301 & 0.000 & 0.329 & $<0.001$ \\
\hline $\mathrm{TC}$ & 0.229 & $<0.001$ & 0.228 & $<0.001$ \\
\hline TG & 0.461 & $<0.001$ & 0.480 & $<0.001$ \\
\hline $\mathrm{HDL}$ & -0.325 & $<0.001$ & -0.318 & $<0.001$ \\
\hline LDL & 0.257 & $<0.001$ & 0.302 & $<0.001$ \\
\hline GGT & 0.448 & $<0.001$ & 0.399 & $<0.001$ \\
\hline AKP & 0.087 & $<0.001$ & 0.334 & $<0.001$ \\
\hline MSTOTAL & 0.474 & $<0.001$ & 0.424 & $<0.001$ \\
\hline $\begin{array}{l}\text { FLI: fatty liv } \\
\text { aminotrans } \\
\text { plasma glu } \\
\text { cholesterol; }\end{array}$ & $\begin{array}{l}\text { : systolic blood press } \\
\text { aspartate aminotran } \\
\text { acid; TC: total chole }\end{array}$ & $\begin{array}{l}\text { blood pre } \\
\text { ne phosp } \\
\text { de; HDL: }\end{array}$ & $\begin{array}{l}\text { max index; WC: wai } \\
\text { ma-glutamyl transf } \\
\text { rotein cholesterol; L }\end{array}$ & $\begin{array}{l}\text { T: alanine } \\
\text { e; FPG: fastin } \\
\text { protein }\end{array}$ \\
\hline
\end{tabular}

\section{Prevalence of different degrees of NAFLD in different age groups}

Because of the difference of sexual prevalence between men and women, this study analyzed the prevalence of NAFLD in different age groups of men and women respectively. In male, the distribution of NAFLD prevalence was different in different age groups, and the prevalence of NAFLD were higher in the $44-59,60-74$ and $\geq 75$ age groups $(=331.014, P<0.001)$. The results were shown in Table.2 and Figure.1a. 
In the female subjects, the distribution of NAFLD prevalence was also different in different age groups, and the prevalence of NAFLD were higher in the 60-74 and $\geq 75$ age groups (= 1189.514, $P<0.001)$. The results were shown in Table. 2 and Figure.1b.

\section{Prevalence of metabolic syndrome in different age groups}

The cumulative prevalence of hypertension, diabetes, obesity and hypertriglyceridemia was $38.2 \%, 30.8 \%, 74.4 \%$ and $50.4 \%$ respectively in male NAFLD patients, while it was $38.6 \%, 14.9 \%, 32.1 \%$ and $17.0 \%$ respectively in male non-NAFLD subjects. The prevalence of diabetes, obesity and hypertriglyceridemia in male NAFLD patients was higher than that in male non-NAFLD subjects $(P<0.05)$. There was no significant difference in hypertension between male NAFLD patients and non-NAFLD subjects $(P>0.05)$ (Table.3). The prevalence of metabolic syndrome in all male age groups is shown in Figure.2a.

The cumulative prevalence of hypertension, diabetes, obesity and hypertriglyceridemia was $36.8 \%, 31.8 \%, 86.6 \%$ and $45.6 \%$, respectively, in female NAFLD patients, while it was $36.2 \%, 9.3 \%, 48.3 \%$ and $12.5 \%$, respectively, in female non-NAFLD subjects. The prevalence of diabetes, obesity and hypertriglyceridemia in female NAFLD patients was higher than that in female non-NAFLD subjects $(P<0.05)$. There was no significant difference in hypertension between female NAFLD patients and non-NAFLD subjects $(P>0.05)$ (Table.3). The prevalence of metabolic syndrome in all female age groups is shown in Figure.2b.

\section{Risk factors associated with the severity of NAFLD}

All subjects were divided into four groups: non-NAFLD group, mild NAFLD group, moderate NAFLD group and severe NAFLD group. Spearman correlation analysis was conducted to analyse the risk factors of NAFLD.

In the male population, the correlation coefficients of FLI, body weight, WC, ALT, UA, TG, GGT and metabolic syndrome with the severity of NAFLD were $0.605,0.531,0.507,0.471,0.301$ and 0.461 , respectively, which were positively correlated with the severity of NAFLD. The correlation coefficients of age, height, systolic blood pressure, diastolic blood pressure, BMI, AST, CR, FPG, TC, LDL and AKP with the severity of NAFLD were $0.128,-0.010,-0.002$, $-0.000,0.266,0.298,-0.029,0.240,0.229,0.257$ and 0.087 , respectively, which had a weak correlation with the severity of NAFLD. The correlation coefficient between HDL and the severity of NAFLD was -0.325 , which was negatively correlated with the severity of NAFLD. The results were shown in detail in Table.4.

In the female population, the correlation coefficients of age, FLI, body weight, WC, ALT, FPG, UA, TG, LDL, GGT, AKP and metabolic syndrome with the severity of NAFLD were $0.363,0.567,0.502,0.545,0.388,0.313,0.329,0.480,0.302,0.399,0.334$ and 0.424 , respectively. The above indexes were positively correlated with the severity of NAFLD. The correlation coefficients of height, systolic pressure, diastolic pressure, BMI, AST, CR and TC with the severity NAFLD were $-0.127,0.020,0.005,0.209,0.237,0.005$ and 0.228 , respectively, which were weakly correlated with the severity of NAFLD. The correlation coefficients between HDL and the severity of NAFLD were -0.318 , which was negatively correlated with the severity of NAFLD. The results were shown in detail in Table.4.

\section{Discussion}

NAFLD has become the most common chronic liver disease in the world. Studies have reported that the prevalence of NAFLD is $24-46 \%$ in western countries[12-14]. Recent studies have reported that the prevalence of NAFLD exceeded that of viral hepatitis, making it the most common liver disease in Asia. The prevalence of NAFLD in Asia is widely variable, ranging from $7.9 \%$ in Indonesia to $54.0 \%$ in the elderly female population in Taiwan[15]. The average prevalence of NAFLD in China was 20.1\%[16]. In the developed cities of Beijing, Shanghai and Hong Kong, the prevalence of NAFLD was $39.5 \%, 43.2 \%$ and $42 \%$, respectively $[4,17-19]$. However, these findings might have some bias due to the study selection bias. A large-scale epidemiological investigation of NAFLD is still lacking in China. Therefore, we conducted a large cross-sectional survey to study the prevalence of NAFLD in Shanghai. Our results showed that the prevalence of NAFLD was $45.55 \%$ in Shanghai, $62 \%$ in males and $27.54 \%$ in females. The prevalence of NAFLD is continuously increasing. It was reported that the global prevalence of NAFLD increased from 15-25\% in five years (2005-2010)[2]. The prevalence of NAFLD in the United States had increased from 5.5\% in 1988-1994 to $21.2 \%$ in 2014[20, 21]. It increased by about $18 \%$ from 1989 to 1998 in Japan[22]. The prevalence of NAFLD in Hong Kong increased by $13.5 \%$ in 3-5 years[23]. In our previous research, we found that the prevalence of NAFLD in the Shanghai working population was 38.17\% in 2012[5]. In this study, we found that the prevalence of NAFLD in the Shanghai working population increased to $41.72 \%$. Recently, a Markov model based on an international multi-centre study was established to assess the progress of NAFLD and NASH. It predicted that the prevalence of NAFLD in all countries will grow at a moderate level (0-30\%) in 2016-2030 with the increase of population and the ageing of population. Urbanization will lead to the highest growth of NAFLD in China, with a growth rate of 29.1\%[24].

The prevalence of NAFLD shows obvious regional differences, which are closely related to the local economic development level and people's lifestyle (sedentary or low physical activity, high-fat and high-sugar food intake, etc.). Studies have pointed out that the prevalence of NAFLD is lower in poor or emaciated people, in those with long-term physical activity, or in those living in rural areas[25]. However, the prevalence of NAFLD in Shanghai is higher than that in the total population in China due to Shanghai's high level of economic development.

NAFLD can occur in any age group. Ageing is an important risk factor for the increasing prevalence of NAFLD[26]. Although most studies were conducted among people at 30-70 years old, the general trend was that the incidence of NAFLD in males peaked at 50-60 years old [27], while the prevalence of NAFLD in females increased with increasing age, especially after menopause[28]. Our research suggested that the prevalence of NAFLD 
varies among different age groups. The prevalence of NAFLD in males was higher in the 44-59 years age group, 60-75 years age group and over 75 years age group; and peaked in the 44-59 years age group. The prevalence of NAFLD in females was higher in the 60-75 years and over 75 years age groups. In this study, we further classified NAFLD into mild, moderate and severe fatty liver. Statistical analysis showed that the proportion of mild to severe NAFLD increased significantly with increasing age.

Being male is also a risk factor for NAFLD[26]. A number of studies have shown that the prevalence of NAFLD in males is higher than in females before the age of $50[26,27,29]$. Our study suggests that the prevalence of NAFLD in males is significantly higher than that in females in people younger than 60 years old. The lower prevalence of NAFLD in young and middle-aged females may be related to the protective effect of female oestrogen.

Obesity, diabetes, hyperlipidaemia and hypertension are also risk factors for NAFLD. In Asia-Pacific Region, the prevalence of NAFLD in obese people ranges from $10-80 \%[30]$. The increase of BMI was positively correlated with the incidence of NAFLD[31]. The prevalence of NAFLD in type 2 diabetes mellitus ranges from approximately 50-75\%[32]. Our study showed that the cumulative prevalence of diabetes, obesity and hypertriglyceridemia of NAFLD patients was higher than that of non-NAFLD normal people, but that there was no significant difference in hypertension between the two groups. There was no significant difference in the prevalence of hypertension between NAFLD patients and normal people. This may be related to the early diagnosis and treatment, good compliance, and good blood pressure control of hypertension patients in Shanghai, which may lead to no impact on the progress of NAFLD.

There are many studies on the risk factors related to NAFLD, but there are no data on the risk factors related to the severity of NAFLD at present. In this study, we further analysed the risk factors related to the severity of NAFLD by Spearman correlation analysis. The results showed that FLI, body weight, WC, ALT, UA, TG, GGT and metabolic syndrome were positively correlated with the severity of NAFLD in the male population. Age, height, systolic pressure, diastolic pressure, BMI, AST, CR, FPG, TC, LDL and AKP were weakly correlated with the severity of NAFLD. HDL was negatively correlated with the severity of NAFLD. In the female population, age, FLI, body weight, WC, ALT, FPG, UA, TG, LDL, GGT, AKP and metabolic syndrome were positively correlated with the severity of NAFLD. Height, systolic pressure, diastolic pressure, BMI, AST, CR and TC were weakly correlated with the severity of NAFLD. HDL was negatively correlated with the severity of NAFLD.

Our research had some limitations. First, we used abdominal ultrasonography to diagnosis fatty liver. The sensitivity of the results is poor, and it was also related to the level of operation of the examiner. Second, we used a large-scale population census, which excluded the fatty liver patients with drug-induced liver damage due to the increase in drug use in the elderly population, and may have underestimated the prevalence of NAFLD in the elderly population. Moreover, in a large-scale population screening, the cost of rheumatism- and autoimmune liver disease related-indicators is high, which is not suitable for all population screenings, and may not completely exclude some patients with autoimmune liver disease.

In conclusion, the prevalence of NAFLD in Shanghai is high. Multiple factors are related to the incidence of NAFLD and the severity of NAFLD. Prevention and early intervention of NAFLD may have great social and economic benefits.

\section{Declarations}

\section{Conflicts of interest}

There are no competing interests to declare.

\section{Funding}

The research was supported by grants from the National Key Research and Development Program of China (No: 2018YFC2002000), the National Natural Science Foundation of China (No: 81701374), Shanghai Medical Leadership Training Program (No: 2019LJ09), Shanghai Municipal Science and Technology Committee Program (No: YDZX20173100004026), Young Excellent Medical Talents Training Program of Health Department of Shanghai Municipal Government (No: 2018YQ58).

\section{Acknowledgments}

We acknowledge the National Natural Science Foundation of China, the Health Department of Shanghai Municipal Government, and the Science and Technology Commission of Shanghai of their funding. We also acknowledge the sonologists and all the members of the gastroenterologists of Huadong Hospital.

\section{References}

1. Calzadilla BL, Adams LA. The Natural Course of Non-Alcoholic Fatty Liver Disease. Int J Mol Sci 2016;17.

2. Younossi ZM, Koenig AB, Abdelatif D, Fazel Y, Henry L, Wymer M. Global epidemiology of nonalcoholic fatty liver disease-Meta-analytic assessment of prevalence, incidence, and outcomes. Hepatology. 2016;64:73-84.

Page $7 / 10$ 
3. Li Z, Xue J, Chen P, Chen L, Yan S, Liu L. Prevalence of nonalcoholic fatty liver disease in mainland of China: a meta-analysis of published studies. J Gastroenterol Hepatol. 2014;29:42-51.

4. Zhai HL, Wang NJ, Han B, et al. Low vitamin D levels and non-alcoholic fatty liver disease, evidence for their independent association in men in East China: a cross-sectional study (Survey on Prevalence in East China for Metabolic Diseases and Risk Factors (SPECT-China)). Br J Nutr. 2016;115:1352-9.

5. Hu X, Huang Y, Bao Z, et al. Prevalence and factors associated with nonalcoholic fatty liver disease in Shanghai work-units. BMC Gastroenterol. 2012;12:123.

6. Chobanian AV, Bakris GL, Black HR, et al. The Seventh Report of the Joint National Committee on Prevention, Detection, Evaluation, and Treatment of High Blood Pressure: the JNC 7 report. JAMA. 2003;289:2560-72.

7. Report of the expert committee. on the diagnosis and classification of diabetes mellitus. Diabetes Care. 2003;26(Suppl 1):5-20.

8. Executive Summary of The Third Report of The National Cholesterol Education Program (NCEP). Expert Panel on Detection, Evaluation, And Treatment of High Blood Cholesterol In Adults (Adult Treatment Panel III). JAMA. 2001;285:2486-97.

9. Tan CE, Ma S, Wai D, Chew SK, Tai ES. Can we apply the National Cholesterol Education Program Adult Treatment Panel definition of the metabolic syndrome to Asians? Diabetes Care. 2004;27:1182-6.

10. Farrell GC, Chitturi S, Lau GK, Sollano JD. Guidelines for the assessment and management of non-alcoholic fatty liver disease in the Asia-Pacific region: executive summary. J Gastroenterol Hepatol. 2007;22:775-7.

11. Fan JG, Jia JD, Li YM, et al. Guidelines for the diagnosis and management of nonalcoholic fatty liver disease: update 2010: (published in Chinese on Chinese Journal of Hepatology 2010; 18:163-166). J Dig Dis 2011;12:38-44.

12. Browning JD, Szczepaniak LS, Dobbins R, et al. Prevalence of hepatic steatosis in an urban population in the United States: impact of ethnicity. Hepatology. 2004;40:1387-95.

13. Williams CD, Stengel J, Asike MI, et al. Prevalence of nonalcoholic fatty liver disease and nonalcoholic steatohepatitis among a largely middleaged population utilizing ultrasound and liver biopsy: a prospective study. Gastroenterology. 2011;140:124-31.

14. Caballeria L, Pera G, Auladell MA, et al. Prevalence and factors associated with the presence of nonalcoholic fatty liver disease in an adult population in Spain. Eur J Gastroenterol Hepatol. 2010;22:24-32.

15. Seto WK, Yuen MF. Nonalcoholic fatty liver disease in Asia: emerging perspectives. J Gastroenterol. 2017;52:164-74.

16. Li Z, Xue J, Chen P, Chen L, Yan S, Liu L. Prevalence of nonalcoholic fatty liver disease in mainland of China: a meta-analysis of published studies. J Gastroenterol Hepatol. 2014;29:42-51.

17. Seto WK, Yuen MF. Nonalcoholic fatty liver disease in Asia: emerging perspectives. J Gastroenterol. 2017;52:164-74.

18. Yan J, Xie W, Ou WN, et al. Epidemiological survey and risk factor analysis of fatty liver disease of adult residents, Beijing, China. J Gastroenterol Hepatol. 2013;28:1654-9.

19. Fung J, Lee CK, Chan M, Seto WK, Lai CL, Yuen MF. High prevalence of non-alcoholic fatty liver disease in the Chinese - results from the Hong Kong liver health census. Liver Int. 2015;35:542-9.

20. Younossi ZM, Stepanova M, Afendy M, et al. Changes in the prevalence of the most common causes of chronic liver diseases in the United States from 1988 to 2008. Clin Gastroenterol Hepatol. 2011;9:524-30. :.e1; quiz e60.

21. Schneider AL, Lazo M, Selvin E, Clark JM. Racial differences in nonalcoholic fatty liver disease in the U.S. population. Obesity (Silver Spring). 2014;22:292-9.

22. Kojima S, Watanabe N, Numata M, Ogawa T, Matsuzaki S. Increase in the prevalence of fatty liver in Japan over the past 12 years: analysis of clinical background. J Gastroenterol. 2003;38:954-61.

23. Wong VW, Wong GL, Yeung DK, et al. Incidence of non-alcoholic fatty liver disease in Hong Kong: a population study with paired proton-magnetic resonance spectroscopy. J Hepatol. 2015;62:182-9.

24. Estes C, Anstee QM, Arias-Loste MT, et al. Modeling NAFLD disease burden in China, France, Germany, Italy, Japan, Spain, United Kingdom, and United States for the period 2016-2030. J Hepatol. 2018;69:896-904.

25. Pati GK, Singh SP. Nonalcoholic Fatty Liver Disease in South Asia. Euroasian J Hepatogastroenterol. 2016;6:154-62.

26. Iqbal U, Perumpail BJ, Akhtar D, Kim D, Ahmed A. The Epidemiology, Risk Profiling and Diagnostic Challenges of Nonalcoholic Fatty Liver Disease. Medicines (Basel) 2019;6.

27. Choi SY, Kim D, Kim HJ, et al. The relation between non-alcoholic fatty liver disease and the risk of coronary heart disease in Koreans. Am J Gastroenterol. 2009;104:1953-60.

28. Lazo M, Hernaez R, Eberhardt MS, et al. Prevalence of nonalcoholic fatty liver disease in the United States: the Third National Health and Nutrition Examination Survey, 1988-1994. Am J Epidemiol. 2013;178:38-45.

29. Alam S, Fahim SM, Chowdhury M, et al. Prevalence and risk factors of non-alcoholic fatty liver disease in Bangladesh. JGH Open. 2018;2:39-46.

30. Polyzos SA, Kountouras J, Mantzoros CS. Obesity and nonalcoholic fatty liver disease: From pathophysiology to therapeutics. Metabolism. 2019;92:82-97.

31. Fan JG, Kim SU, Wong VW. New trends on obesity and NAFLD in Asia. J Hepatol. 2017;67:862-73.

Page 8/10 
32. Lee YH, Cho Y, Lee BW, et al. Nonalcoholic Fatty Liver Disease in Diabetes. Part I: Epidemiology and Diagnosis. Diabetes Metab J. $2019 ; 43: 31-45$.

Figures

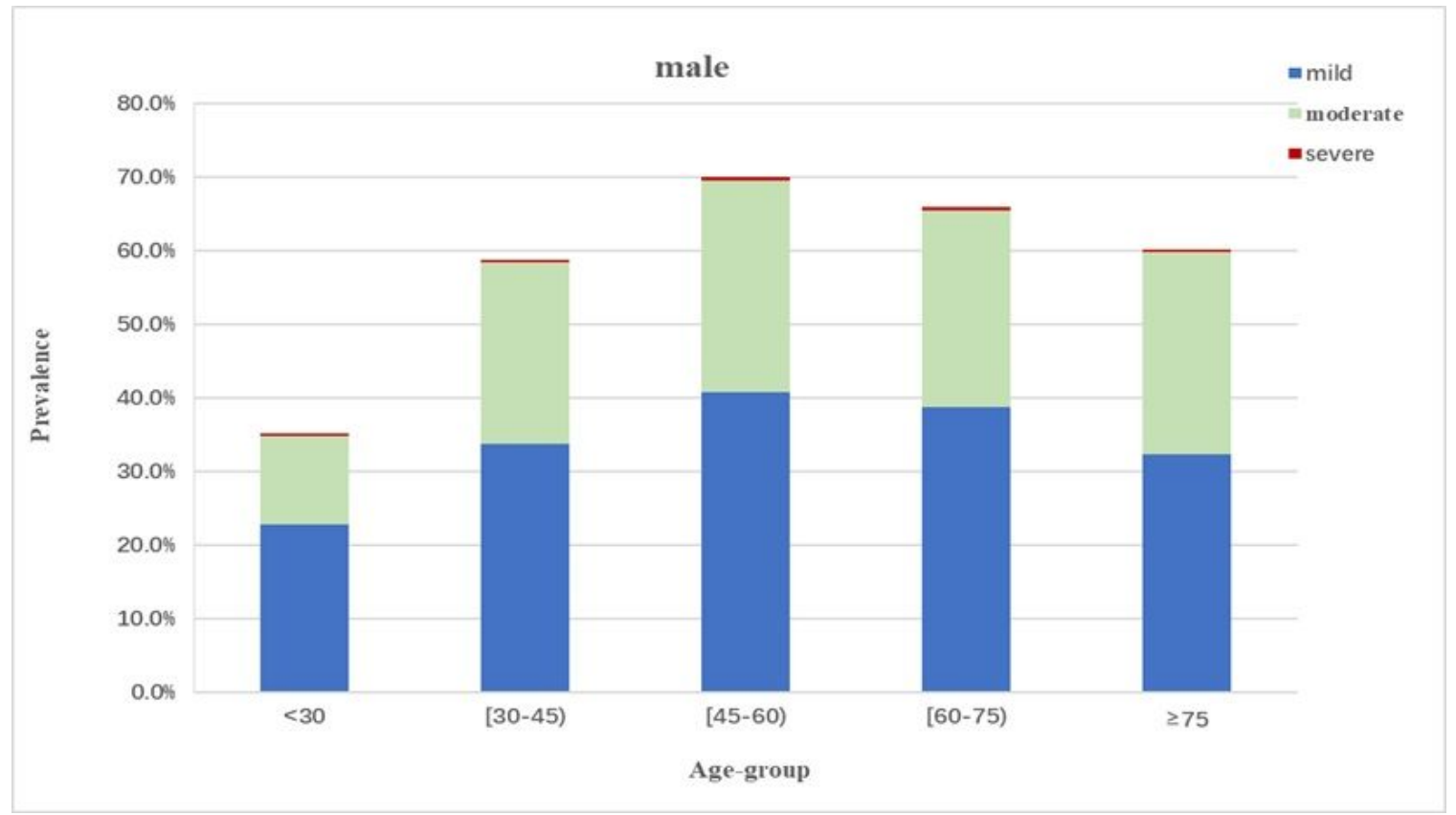

\section{Figure 1}

Age-specific prevalence of NAFLD in males and females 

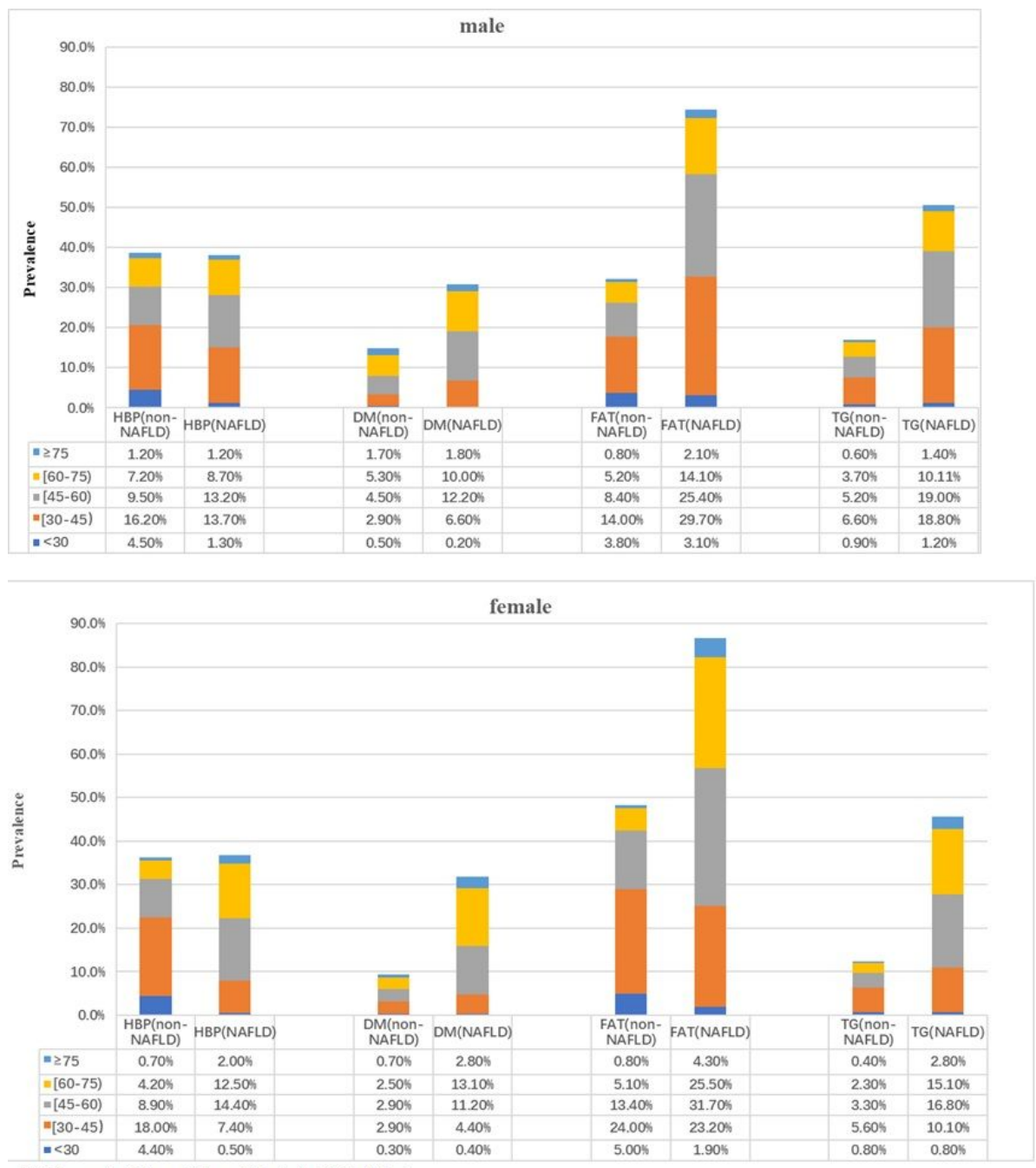

HBD: hypertension; DM: type 2 diaberes; FAT: obesity TG: Dyslipidemis

\section{Figure 2}

Age specific prevalence of metabolic syndrome in male and female 